\title{
STATEMENT
}

\section{We are tired of 'adrenal fatigue'}

\author{
I L Ross, ${ }^{1} \mathrm{MB}$ ChB, FCP (SA), FRCP (London), Cert Endocrinology and Metabolism (SA), PhD (Medicine); J Jones, ${ }^{2}$ BPharm; \\ M Blockman, ${ }^{2}$ BPharm, MB ChB, PG Dip Int Res Ethics, MMed
}

\author{
${ }^{1}$ Division of Endocrinology and Diabetic Medicine, Department of Medicine, Faculty of Health Sciences, University of Cape Town, South Africa \\ ${ }^{2}$ Division of Pharmacology, Department of Medicine, Faculty of Health Sciences, University of Cape Town, South Africa
}

Corresponding author: I L Ross (ian.ross@uct.ac.za)

\begin{abstract}
There are numerous articles in the lay press discussing the existence and under-diagnosis of adrenal fatigue. Proponents of adrenal fatigue state that millions of people have under-active adrenal glands due to repeated stressors, resulting in numerous nonspecific symptoms such as fatigue, insomnia, joint pain and weight gain, among others. In studies to date, proposed methods to assess adrenal fatigue have produced conflicting results and the methodology for assessing the hypothalamic-pituitary-adrenal (HPA) axis has often been inappropriate. Furthermore, only a minority of studies have actually examined the HPA axis. Current evidence does not support the existence of adrenal fatigue or the usefulness of supplements to support adrenal function.
\end{abstract}

S Afr Med J 2018;108(9):724-725. DOI:10.7196/SAMJ.2018.v108i9.13292

\section{The theory}

Adrenal fatigue, according to several online (mainly non-medical) publications, is a little-known condition that doctors dismiss. These publications claim that sufferers from this condition feel constantly tired, and it takes time before this 'diagnosis' is made. Their symptoms include feeling exhausted, irritable and unhappy. It is suggested in the lay press that the adrenal glands in these people function below the normal threshold, with the result that individuals feel tired and stressed. The internet is inundated with claims from people that they suffer from adrenal fatigue. As physicians, our responsibility is to determine whether a true medical disorder exists, and whether an underlying sinister condition is being overlooked. Many patients resort to taking untested supplements or unknown formulations and often consult alternative medical practitioners who reinforce and promote the existence of adrenal fatigue.

Adrenal fatigue is said to occur when the adrenal glands become drained and unable to produce cortisol. It is a label used to describe a wide range of vague symptoms including fatigue, insomnia, 'brain fog', joint pain, allergies and weight gain. This is by no means a comprehensive list of all possible symptoms attributed to adrenal fatigue. Proponents of the existence of adrenal fatigue suggest that it affects hundreds of millions of people globally and that the adrenal glands, while not failing, cannot keep pace with the demands placed on them. It is supposed to denote a condition in which repeated exposure to stressful conditions results in depletion of the adrenal glands with resultant functional failure, giving rise to the feeling of fatigue. There are no confirmed diagnostic and special investigations on how to screen for adrenal fatigue, and there is no ICD-10 code delineating it as a recognised clinical disorder.

\section{Assessment of adrenal function}

In critical illness, the adrenal glands are submaximally stimulated and frequently do not mount the incremental cortisol response of $250 \mathrm{nmol} / \mathrm{L}$ following the synthetic adrenocorticotrophic hormone $(\mathrm{ACTH})$ stimulation test. This is termed relative adrenal insufficiency. ${ }^{[1]}$ The diagnosis of Addison's disease or true adrenal failure is confirmed by the simultaneous measurement of earlymorning serum cortisol and plasma ACTH. In equivocal cases, the standard $250 \mu \mathrm{g}$ synthetic ACTH stimulation test is used to definitively diagnose Addison's disease and distinguish it from normal adrenal gland function. ${ }^{[2]}$

\section{Assessment of adrenal fatigue}

Various publications on the available methods to assess adrenal fatigue and the hypothalamic-pituitary-adrenal (HPA) axis report contradictory results, and in most cases the most appropriate methodology to assess the HPA axis was not utilised. ${ }^{[3]}$ The usual test to diagnose adrenal insufficiency is the synthetic ACTH stimulation test. Yet only a minority of studies utilised the synthetic ACTH test, and when these tests were performed, they often used lower than recommended doses. Moreover, counterintuitively the synthetic ACTH stimulation test showed a greater rise in cortisol in presumed sufferers from fatigue than in healthy control subjects. ${ }^{[4]}$

There are several thousand PubMed articles referring to adrenal fatigue, but only 13 have examined the HPA axis. A recent systematic review of adrenal fatigue has recommended that prospective studies should correlate symptoms with a standard-dose synthetic ACTH stimulation test. ${ }^{[5]}$

Alternative practitioners have used various methods to attempt to prove the existence of adrenal fatigue, often at great expense, with no proven validity. The cortisol awakening response, used extensively in the literature to document psychological stress, was one of the methods to diagnose adrenal fatigue with diminished response on waking. The salivary cortisol rhythm as the measure between the change in cortisol between morning, afternoon and late night has also been utilised. Two-thirds of participants showed no difference in salivary cortisol between healthy control subjects and those who were fatigued. ${ }^{[6]}$ Several investigators have proposed late-night cortisol, but this has been found in some studies to be lower, similar or higher in individuals identified as suffering from adrenal fatigue compared with controls, bringing its reliability into question. Naturopaths have utilised the whole-day salivary cortisol area under the curve to diagnose adrenal fatigue, but this has demonstrated conflicting data and the inherent variability of this test precludes its reliability. Dehydroepiandrosterone sulphate (DHEAS), a marker of adrenal androgen production, has been utilised by some studies to indicate 
adrenal fatigue by virtue of its lower than normal concentrations. However, the correlation of DHEAS with symptoms in supposed sufferers has been inconsistent. ${ }^{[7]}$

Ashwagandha, rhodiola, liquorice root, ginseng, schizandra and maca are sold in order to support adrenal function, with no objective benefit for this indication. With impaired test results, unfortunately corticosteroids are often prescribed with subjective symptom improvement. Although corticosteroids may impart a euphoric effect and raise exercise tolerance in healthy individuals, they have a considerable side-effect profile. Without data for their benefit in adrenal fatigue, any risks would be unacceptable.

In our setting in South Africa, the various products listed in the paragraph above are available. The conservative accumulative costs for these agents are approximately ZAR1 200 per month, with no proven benefit and unknown harms.

\section{Conclusions}

The Endocrine Society of the USA has issued a warning statement that adrenal fatigue is not a medical condition, and a systematic review has indicated that adrenal fatigue does not exist. ${ }^{[5]}$ It has been proposed that if patient fatigue exists, true hypoadrenalism should be definitively excluded, as Addison's disease is associated with high mortality.

Until there is objective, reproduceable clinical evidence for its diagnosis, adrenal fatigue does not exist, and patients should be appropriately investigated and managed for their symptoms.

\section{Acknowledgements. None.}

Author contributions. ILR conceptualised the article, with MB and JJ contributing to the writing and integrity. All had equal eventual contributions.

Funding. None.

Conflicts of interest. None.

1. Boonen E, van den Berghe G. Mechanisms in endocrinology: New concepts to further unravel adrenal insufficiency during critical illness. Eur J Endocrinol 2016;175(1):R1-R9. https://doi.org/10.1530/EJE15-1098

2. Husebye ES, Allolio B, Arlt W, et al. Consensus statement on the diagnosis, treatment and followup of patients with primary adrenal insufficiency. J Intern Med 2014;275(2):104-115. https://doi. $\operatorname{org} / 10.1111 /$ joim. 12162

3. Cleare AJ. The HPA axis and the genesis of chronic fatigue syndrome. Trends Endocrinol Metab 2004;15(2):55-59. https://doi.org/10.1016/j.tem.2003.12.002

4. Wolfram M, Bellingrath S, Feuerhahn N, Kudielka BM. Emotional exhaustion and overcommitment to work are differentially associated with hypothalamus-pituitary-adrenal (HPA) axis responses to a low-dose ACTH1-24 (Synacthen) and dexamethasone-CRH test in healthy school teachers. Stress 2013;16(1):54-64. https://doi.org/10.3109/10253890.2012.683465

5. Cadegiani FA, Kater CE. Adrenal fatigue does not exist: A systematic review. BMC Endocr Disord . Cadegiani FA, Kater CE. Adrenal fatigue does not exist: A s
2016;16(1):48. https://doi.org/10.1186/s12902-016-0128-4

6. Mommersteeg PM, Heijnen CJ, Verbraak MJ, van Doornen LJ. Clinical burnout is not reflected in the cortisol awakening response, the day-curve or the response to a low-dose dexamethasone suppression test. Psychoneuroendocrinology 2006;31(2):216-225. https://doi.org/10.1016/j.psyneuen.2005.07.003

7. Sonnenschein M, Mommersteeg PM, Houtveen JH, Sorbi MJ, Schaufeli WB, van Doornen LJ. Exhaustion and endocrine functioning in clinical burnout: An in-depth study using the experience sampling method. Biol Psychol 2007;75(2):176-184. https://doi.org/10.1016/j.biopsycho.2007.02.001

Accepted 9 April 2018 